\title{
Experimental study of freak wave impacts on a tension-leg platform
}

\author{
Min Luo ${ }^{\mathrm{a}, \mathrm{b}}$, Chan Ghee $\mathrm{Koh}^{\mathrm{c}}$, Wei Xian Lee ${ }^{\mathrm{c}}$, Pengzhi Lin ${ }^{\mathrm{d}, *}$, Dominic E. Reeve ${ }^{\mathrm{b}}$ \\ a Ocean College, Zhejiang University, Zhoushan 316021, Zhejiang, China \\ ${ }^{\mathrm{b}}$ Zienkiewicz Centre for Computational Engineering, College of Engineering, Swansea University, Swansea SA1 8EN, \\ United Kingdom \\ ${ }^{c}$ Department of Civil and Environmental Engineering, National University of Singapore, Singapore 117576 \\ d State Key Laboratory of Hydraulics and Mountain River Engineering, Sichuan University, Chengdu 610065, China
}

\section{Abstract}

This study investigates the freak wave impinging on a tension-leg platform through wave flume experiments. The freak waves are generated using the focused wave theory. By adjusting the wave focusing location, different incident wave scenarios at the structure location are produced. Simultaneous measurements of wave shape evolutions upon impingement, wave impact pressures on the platform deck, platform motions and tether forces are carried out for synchronized analyses of the wave kinematics/dynamics and structural responses. The variation of these parameters with the incident wave profile is studied. It is found that although applying less intensive local impact pressures as compared to the highly-breaking freak wave, the slightly-breaking or non-breaking freak wave imposes the same level of adverse effect on the platform's global stability in terms of motions and tether forces. In addition, the high-crest freak wave causes violent motions of the floating platform, which are likely to induce snap loads of large amplitude and high occurrence frequency in tethers. The published results would provide useful benchmarks for validating numerical and analytical models.

Keywords: freak wave; tension-leg platform; wave impact; platform motion; tether force

\section{Introduction}

Freak waves, also called rogue waves or monster waves, are characterized by much higher wave heights than those expected for the sea state (more than twice of the significant wave height) [1], and can appear surprisingly from nowhere in the ocean areas of arbitrary water depth [2]. Freak waves may become unstable and overturn/break as a plunging breaker. When attacking marine structures like the ship, oil/gas platform and marine renewable energy device, the freak wave can induce catastrophic damages to these structures [1-3]. Field measurements of freak waves include the Draupner wave with a maximum crest of $18.49 \mathrm{~m}$ and the Andrea wave with a maximum crest of $14.97 \mathrm{~m}$ [4]. The industry and research communities have highlighted the occurrence of the freak wave and the severity of the associated hazards to marine structures, as well as raised the importance of taking freak waves into account in design [5]. However, until now there has been little consensus on the physics and probability

\footnotetext{
* Corresponding author.

E-mail: cvelinpz@126.com (P. Lin).
} 
of occurrence of freak waves [5]. Besides, limited data about freak wave interaction with floating structures are documented in the literature. In this context, this experimental work studies freak waves impinging on a tension-leg platform to examine the platform's hydrodynamic performance under such a low-probability high-consequence event and provides high-quality data for validating numerical and analytical models.

The large wave crest of a freak wave is caused by the local concentration of wave energy, which can be produced by the wave refraction in varying seabed topography and the dispersive focusing of a frequency-modulated wave packet [1]. Based on this principle, the freak wave has been generated successfully in physical and numerical wave basins through linear wave superposition [6-10] or solving the nonlinear Schrödinger equation to consider the wave nonlinearity [11, 12]. With the successful wave generation, some studies have examined the freak wave actions on marine and coastal structures. A freak wave may evolve into a plunging breaker, which causes intensive impact loads among the various wave-structure interaction scenarios. Therefore, the plunging-type freak wave has always been studied as a representative of the freak wave [8].

Various ocean/marine structures have vertical faces. Hence many researchers studied the freak wave impacts on fixed vertical walls experimentally $[6,13]$ and numerically $[14,15]$. It was found that the wave impact characteristics are closely related to the incident wave kinematics (i.e. the wave profile upon wave impact happens). Specifically, Bullock, Obhrai [13] classified four types of impact with distinct features, i.e. slightly-breaking wave impact, low-aeration impact, high-aeration impact and broken wave impact. The dynamic coupling of entrapped air and impinging wave influences the local impact pressure notably. However, the documented observations are contradictory. Some researchers found that the magnitude of impact pressure decreases and the pressure rise time increases with the amount of entrapped air [6, 13], but others got the opposite findings regarding pressure amplitude [16]. The disagreement is attributed to the complicated physics of water-air interaction.

Substantial studies examined the violent wave impact on cylinders that mimics the foundation/pier of marine structures [17-19]. The wave slamming load [9, 20-22] and green water [23-25] on fixed deck structures of simplified shapes have also been the subjects of extensive studies. It has been pointed out that the forces or green water induced by freak or plunging waves are much intense than those caused by equivalent-sized regular or sea-state irregular waves, highlighting the necessity to examine the freak wave actions. Besides, it was found that the wave kinematics and impact pressures are closed related to the impact condition (i.e. incident wave profile).

The research work tackling wave impact on fixed structures is massive in the literature as discussed above. However, there are not too many documented studies on extreme wave interaction with floating structures, which represent a broad range of applications in marine engineering such as floating breakwater, semi-submersible platform and tension-leg platform (TLP). It is challenging to numerically simulate or experimentally measure the highly nonlinear breaking waves, the water-air interaction due 
to air entrapment under certain conditions and the dynamic fluid-structure coupling. Documented studies include those focused on floating structures of simplified shapes such as moored or hinged boxshape floating bodies [26-28]. For the semi-submersible platform that has been applied extensively in oil/gas exploitations and marine renewable energy devices, the vortex-induced motions and wave forces under a range of regular and irregular wave conditions have been investigated [29, 30]. In addition, the influence of the wave group characteristics of a series of extreme wave sequences on semisubmersible's motion was studied [31]. For TLP, the freak wave actions were simulated using the Smoothed Particle Hydrodynamics in [32] and [33], which examined the influences of incident wave angle and mooring system configuration on platform responses, respectively. However, no quantitative validations of the numerical results were made probably due to the lack of relevant data. The green water on a tensionleg-moored box-shape deck was studied experimentally in [34] and it was found that the green-water induced wave crest above the deck at the initial stage was much higher than that on a fixed platform. Based on the same experimental setup, Chuang, Chang [35] investigated the plunging wave impacts and highlighted the cushioning effect in reducing the impact pressure. In these two studies, the measurements included wave impact pressures, fluid velocities, void fractions and structural motions, but the forces in the tethers were not considered. More recently, the wave-in-deck impacts on fixed [36] and floating [37] TLP models under the wave-spectrum-governed sea state in cyclonic conditions have been studied experimentally. The wave impact pressure, as well as the tether forces and surge motion for the floating model, were measured and their correlations were investigated.

Although significant progress has been made in revealing the hydrodynamic properties of tensionleg platforms, the problems remain to be solved or complemented include: (1) how the wave impact pressures and platform responses behave under the actions of freak waves; and (2) how the impact condition (or incident wave profile) of a freak wave affects the impact pressures and platform responses.

This study investigates the hydrodynamic properties of a TLP model recently proposed in a new design through carefully controlled wave flume experiments. A scaled freak wave that has similar properties to the Draupner wave was considered, aiming to address the aforementioned two questions. Comprehensive and high-fidelity measurements were conducted, including the highly deformed wave profiles during the wave impingement process, impact pressures on upper deck walls, motions of the platform, and forces in the tethers. The characteristics of the wave impact pressures and structural responses as well as their correlations with the incident wave profile are discussed. The experimental data provide important benchmarks for model validation/calibration. In the following, the experimental methodology will be introduced in Section 2. The results and discussion are detailed in Section 3 followed by a repeatability analysis of the data in Section 4 . This study is concluded in Section 5. 


\subsection{Experimental model setup}

The experiments were conducted in the ferrocement wave flume $(36 \mathrm{~m} \times 2 \mathrm{~m} \times 1.3 \mathrm{~m})$ in the Hydraulic Engineering Laboratory at the National University of Singapore. Fig. 1 shows the schematic view of the experimental setup. The wave flume is equipped with a single-piston wave maker, being able to generate unidirectional regular and irregular waves. Placed at the location with glass windows ( $\sim 12 \mathrm{~m}$ away from the wave maker) was a scaled TLP model (scale-down factor $\lambda=1 / 75$ based the standard dimensions real TLP models) made of transparent Perspex of $8 \mathrm{~mm}$ thickness. The dimensions of the floating model are shown in Fig. A1 in Appendix A. It contains two pontoons, four columns (of height $0.24 \mathrm{~m}$ and the same width as the pontoons) and a rigid buoyant upper hull deck box of height $0.11 \mathrm{~m}$ (see Fig. $1 \mathrm{~b}$ and Fig. 2). Two horizontal bracings that connect the port and starboard columns were installed to enhance the lateral stability of the platform.

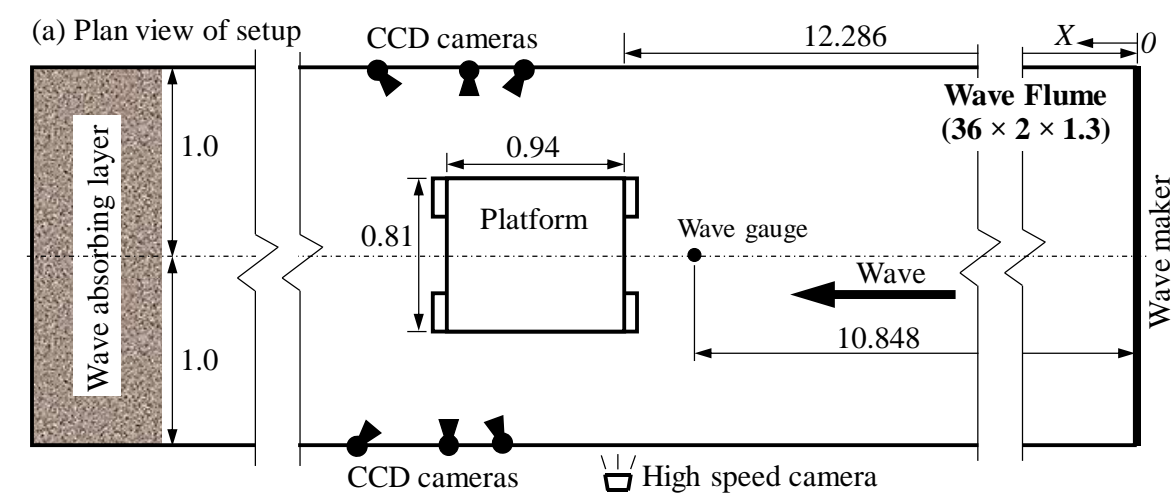

(b) Front view of the floating platform
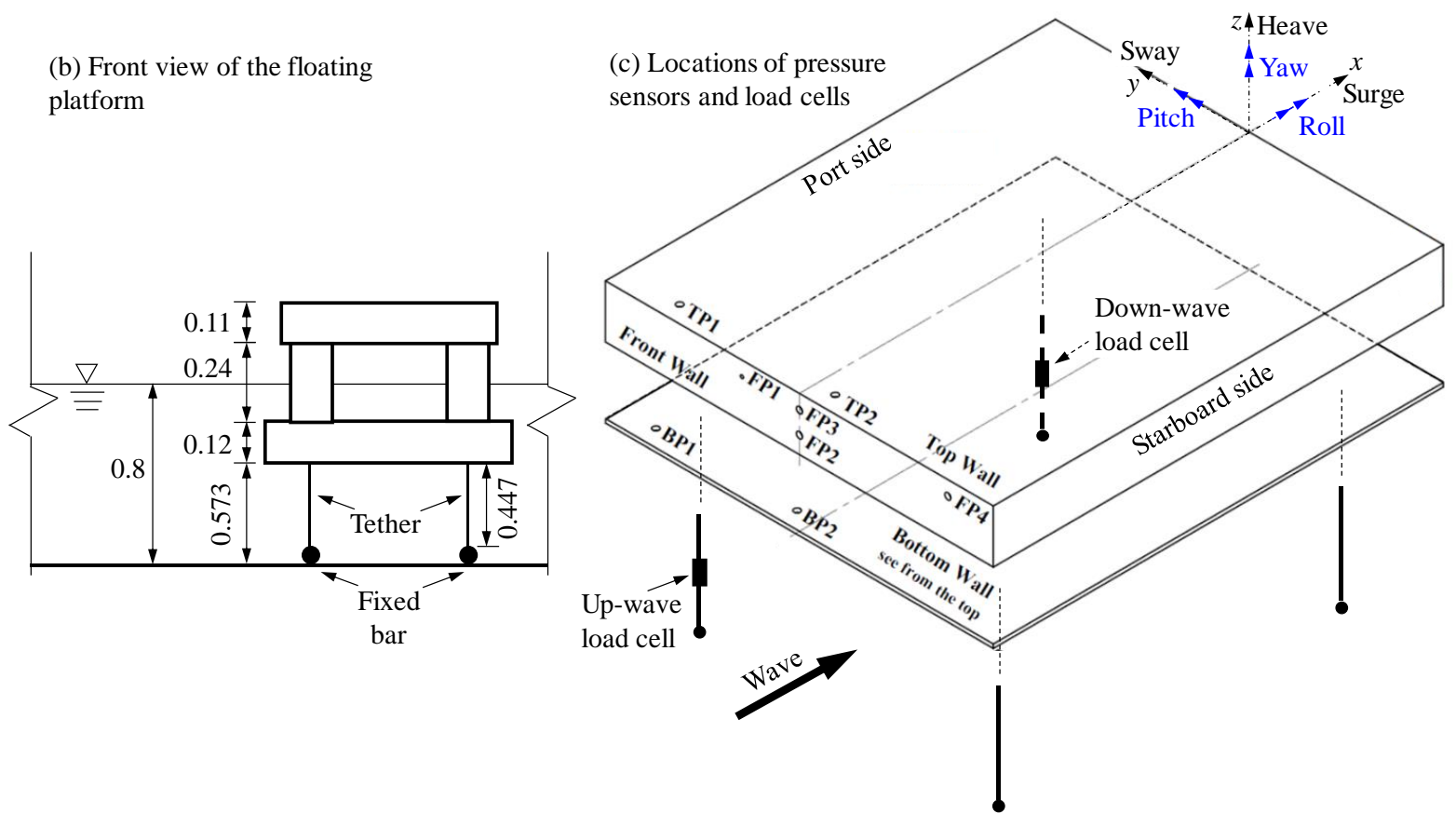

Fig. 1. Schematic view of the experimental setup (Unit: m): (a) plan view of the whole setup; (b) front view of the floating platform; (c) locations of pressure sensors and load cells \& sign convention of the 6-degree-of-freedom motions (the pontoons and columns are omitted for clarity) 
Since the span of the upper deck is relatively large ( $0.81 \mathrm{~m}$ for beam and 0.94 for length), some stiffeners were added inside to enhance the lateral stiffness of the deck walls such that under wave actions the wall deformations are negligible. Some holes were drilled in the upper deck for the installation of pressure sensors. Because of the stiffeners and sensors, some weights were added on the top of the upper deck to ensure the horizontal level of the platform (see Fig. 2a) when it freely floats on water. The platform model was constrained by four adjustable (through a turnbuckle) steel tethers from the bottom of the pontoons. The tethers were aligned with the geometrical centre of each column when viewed in plan. Load cells were installed in the middle of two of the tethers (see Fig. 1c) to measure the tension forces (more details are given in Section 2.2). The bottom ends of the tethers were anchored to two fixed horizontal bars at a height of $0.126 \mathrm{~m}$ from the flume bottom (see Fig. 2b). When the water was stationary, the four tethers were tuned to be of the same length such that the platform was horizontal. The horizontal level was confirmed by the readings of the load cells in a way that the same reading means the platform is horizontal. The tethers are made of steel strand of $1 \mathrm{~mm}$ diameter with Young's modulus of $180 \mathrm{GPa}$ [38] and the length of each tether is $0.447 \mathrm{~m}$. This gives an axial stiffness of $3.163 \times 10^{5} \mathrm{~N} / \mathrm{m}$ (bending and torsional stiffness are negligible because of the small diameter of strand). Under the maximum tension force of $400 \mathrm{~N}$, the elongation of the tether is around $1.265 \mathrm{~mm}$, which is very small.

For ease of discussion, the following convention is adopted in this study. The side of the platform that faces the incident wave is up-wave or upstream and the other end is down-wave or downstream. The direction perpendicular to the wave flume wall is the width (or beam) direction. The load cells were installed on the port side of the platform (on the left of the wave propagation direction). The up-wave vertical wall of the deck is called the front wall to distinguish it from the vertical walls at the port and starboard sides.
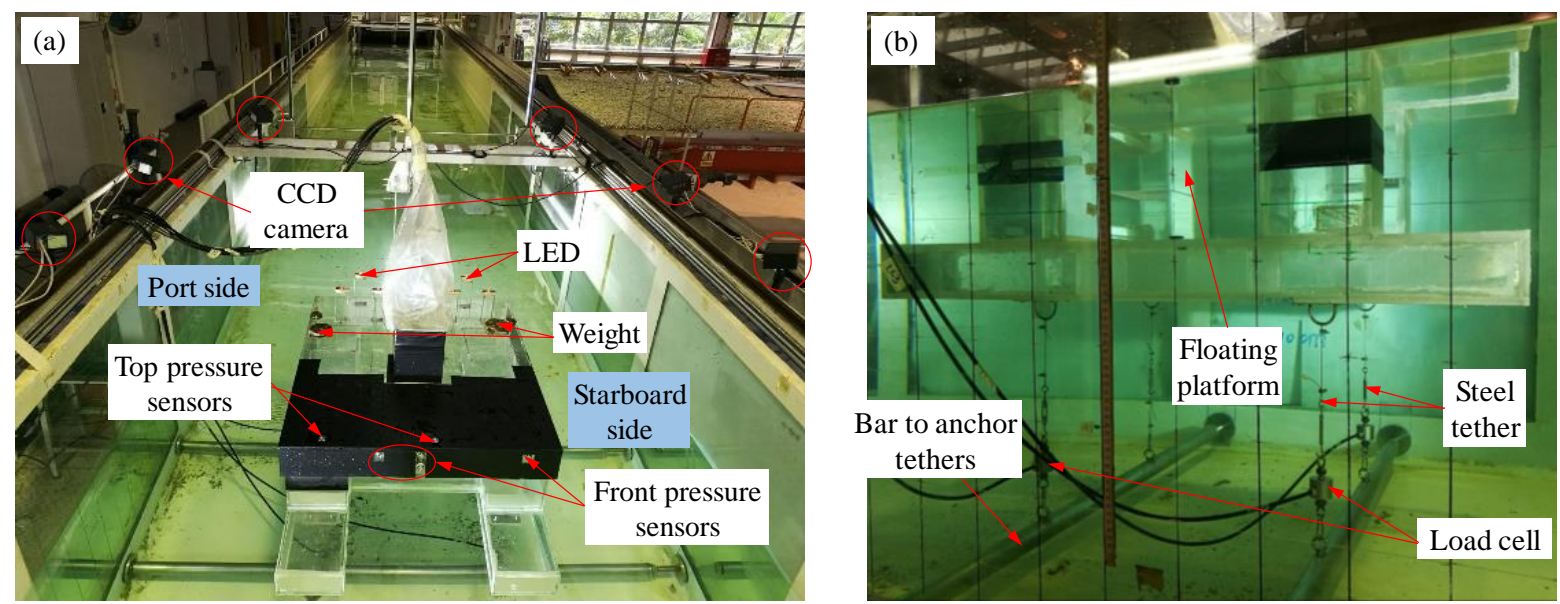

Fig. 2. Model setup photos: (a) general setup; (b) tether and anchor system of the floating platform

One novelty of this study is the comprehensive measurement of multiple parameters of the wavestructure interaction. Firstly, the wave elevation at $1.438 \mathrm{~m}$ in front of the platform ('Wave gauge' in 
Fig. 1a) was measured by a capacitance type wave gauge (KENEK CHT4-60). This location was chosen such that the crest of a mature but non-breaking freak wave was measured (it was difficult to measure the elevation of breaking waves). Secondly, the highly deformed wave profiles during the transit impact process were recorded by a high-speed camera (model Photron FASTCAM SA1.1 using a shutter speed of $1000 \mathrm{fps}$ ) located outside of the glass flume wall (see Fig. 1a). This enables a detailed analysis of the incident wave patterns.

Wave pressures were measured at eight locations by the ATM.1ST analogy pressure sensors (accuracy is $0.1 \%$ full scale and response time less than 1 millisecond). The measuring diaphragm of the sensors is a circle of diameter $4 \mathrm{~mm}$, being larger than those reported in [14, 39]. Although an element of areal averaging may cause the measured pressure to be slightly smaller than the pressure measured over a smaller area [40], the adequacy of the present pressure sensor model in measuring the localized impact pressure was demonstrated in previous studies [9, 41]. The sensor is based on a piezoresistive measuring element and hence can capture the pressure applied on it, irrespective of whether the pressure change is caused by water, air or water-air mixture that occurred in this study. Since the waves considered in this study impacted mainly at the up-wave side of the platform, the pressure sensors were installed at the up-wave side of the upper deck as sketched in Fig. 1c. There were four pressure sensors (FP1, FP2, FP3 and FP4) on the front wall. FP2 and FP3 measured the wave pressures on the centre line at a distance of $35 \mathrm{~mm}$ and $80 \mathrm{~mm}$ from the bottom of the deck, respectively. FP1 and FP4 were at the same elevation as FP3 (i.e. $80 \mathrm{~mm}$ from the deck bottom), locating at $120 \mathrm{~mm}$ of the port side and $310 \mathrm{~mm}$ of the starboard side from the centre line, respectively. Two sensors were used to measure the green water impacts at the top wall of the deck box: TP2 near the centre line and TP1 $285 \mathrm{~mm}$ from the centre at the port side. The wave impact pressures at the bottom wall of the deck structure were measured near the centre line (BP2) and $335 \mathrm{~mm}$ from the centre at the port side (BP1). The pressure sensor locations are detailed in Fig. A2 in Appendix A.

The six-degree-of-freedom platform motions were measured by a PhaseSpace Motion Capture System. The system is composed of a CCD camera array fixed on the flume walls and a LED array attached to the deck of the platform (see Fig. 2). The CCD cameras track the motions of the LEDs, from which the six-degree-of-freedom motions of the platform can be calculated [42]. As the platform moves, restoring forces are generated in the tethers. The forces in the up-wave and down-wave tethers of the port side were measured by DDEN submersible load cells (see Fig. 1c). The actual wave paddle motion was measured by a linear variable differential transformer (LVDT), which could be used for numerical simulations. In experiments, the measurement signals from the LVDT, load cells and pressure sensors were recorded by an oscilloscope with a sampling frequency of $2000 \mathrm{~Hz}$. Hence, these data were synchronized. The synchronization of these data with the platform motion was achieved manually by analysing all the data comprehensively. 
As discussed in the introduction, several strategies have been developed for freak wave generation. However, it is not straightforward to generate freak waves that follow the wave statistics of a certain sea area because the statistical properties of freak waves are still not well understood [5]. To reproduce a down-scaled real freak wave is another option that has been achieved by some researchers [43]. This approach, however, has very stringent requirements on the wave flume and wave maker that could not be met by the wave flume described here. Instead, this study used unidirectional freak waves based on the principle of wave focusing (see Section 2.4). The crest front steepness and full-scale (up-scaled by ג) wave height and crest height of the generated freak wave were $0.34,25.05 \mathrm{~m}$ and $14.4 \mathrm{~m}$, respectively, being close to those of the Draupner wave $(0.43,25.01 \mathrm{~m}$ and $18.49 \mathrm{~m}$, respectively) [4]. The close similarity between the studied freak wave and the documented real one allows the research findings herein to provide some physical insight into the actions of real freak waves [44].

The water depth was selected to be $0.8 \mathrm{~m}$, which is an intermediate one (see Section 2.4). The corresponding full-scale depth is admittedly smaller than the water depths of typical TLPs in operation, due to the constraints of the experimental facilities. The level of the platform was adjusted such that about half of each column was submerged (see Fig. 1b). This represents the operating mode of a real offshore platform, which is the most common state during its service time. Since the oceanic freak wave can happen unexpectedly, this wave condition simulates the sudden attack on a floating platform by a freak wave whose intensity is close to the Draupner wave. While this wave scenario is more severe than the wave conditions currently considered in design codes, there has been an increase in global wave power as a consequence of the oceanic warming $[45,46]$. Therefore, there is a need in the near future to consider more severe waves than the current design for ocean structures.

\subsection{Freak wave generation based on wave focusing}

Freak waves in this study were generated by the focused wave theory [47], which describes the dispersive focusing of a group of wave components (wave packet) with different frequencies. According to this theory, the wave elevation $\eta$ with space $x$ and time $t$ is as follows:

$$
\eta(x, t)=\sum_{i=1}^{N} a_{i} \cos \left[k_{i}\left(x-x_{f}\right)-2 \pi f_{i}\left(t-t_{f}\right)\right]
$$

where $N$ is the number of wave components, and $a_{i}, k_{i}$ and $f_{i}$ the wave amplitude, wave number and wave frequency of the $i$-th wave component, respectively. The wave amplitudes of all the components in a wave packet can be determined in three ways: constant wave amplitude [6]; following a specified wave spectrum [26]; constant wave steepness [48]. The present study adopted the constant-waveamplitude approach that gives a (near) white-noise spectrum of the wave packet. The selection of such

213 a wave condition is adequate for this study focusing on the deterministic analysis of a specific freak wave impinging on a structure over a short duration. The wave frequency $f_{i}$ is discretised uniformly over a frequency band $\left[f_{\min }, f_{\max }\right] . x_{\mathrm{f}}$ specifies the wave focusing location, by adjusting which different 
wave profiles at the structure location can be produced to study the effect of the incident wave profile on wave impact characteristics. $t_{\mathrm{f}}$ is the wave focusing time, which is dependent on $x_{\mathrm{f}}$ and determined such that the initial paddle motion is very close to zero (avoiding the jerk motion of the wave maker).

32 wave components were introduced. The frequency band of the wave components in all the studied cases was selected to be $f_{\min }=0.32 \mathrm{~Hz}$ and $f_{\max }=0.96 \mathrm{~Hz}$. The characteristics (or middle) wave frequency of $0.64 \mathrm{~Hz}$ (period $1.56 \mathrm{~s}$ ) was used to evaluate the properties of the wave packet. This corresponds to a $k \cdot d$ ( $k$ is wave number and $d$ is water depth) number of 1.47 , which is located within the intermediate water depth range. Scaling up (by the square root of $\lambda$ ) the typical wave period to the real sea gives a wave period of $13.5 \mathrm{~s}$, which is within the period range of ordinary ocean waves. The wave amplitude $a_{i}$ was selected to be $0.0068 \mathrm{~m}$. This leads to a wave height of $0.334 \mathrm{~m}$ and a crest height of $0.192 \mathrm{~m}$ in front of the platform (see Fig. 4).

The characteristics of freak wave impacts are highly dependent on the waveform just before the impact happens $[6,13,14]$. To investigate this, a variety of wave impact scenarios that have different incident wave profiles were produced by adjusting the wave focusing location $x_{\mathrm{f}}$ in wave generation. Specifically, three distinct wave impact patterns were classified and studied. With the downward shift of the wave focusing location, they are labelled as pattern 2, pattern 3 and pattern 4, as sketched in Fig. 3. In pattern 2 , the freak wave is fully developed and breaks upon touching the structure, with the tongue of the wave crest first impacting on the front wall of the deck. In pattern 3, the wave also breaks upon touching the structure, but with the tongue of the wave crest first impacting on the top of the deck. In pattern 4 , the height and steepness of the wave are still developing while the wave impact occurs. Pattern 1 is the scenario where the freak wave breaks before reaching the platform and plunges forward to the bulk water, inducing a jet flow that impinges on the platform front and bottom. However, no data were recorded for this wave pattern, which will be examined in future studies. Among the wave patterns, patterns 1 to 3 are characterized as highly-breaking waves, while pattern 4 involves slightly- or nonbreaking waves. All the experimental cases are summarized in Table 1. In what follows, the following labelling convention is adopted: dabxfgpqrs refers to the experiment with $d=0 . a b \mathrm{~m}$ and $x_{\mathrm{f}}=$ gp.qrs $\mathrm{m}$. For example, d80xf12000 represents the case with $d=0.80 \mathrm{~m}$ and $x_{\mathrm{f}}=12.000 \mathrm{~m}$.

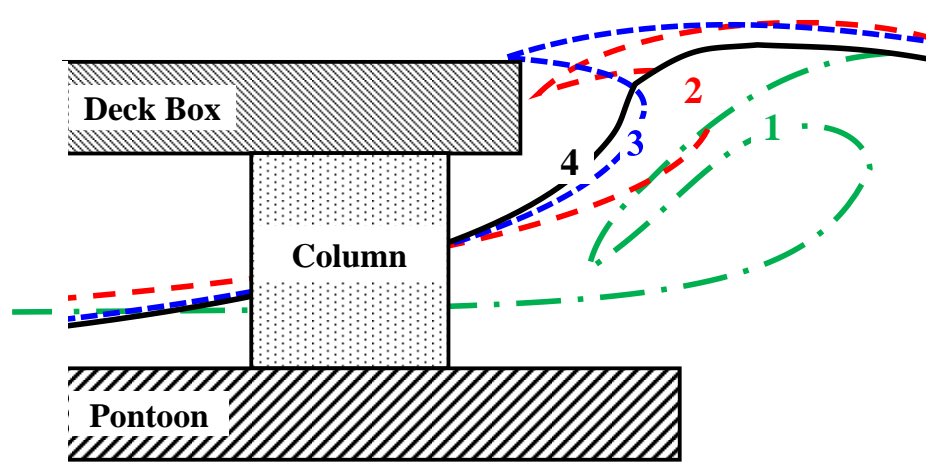

Fig. 3. Schematic view of three wave-impact patterns. Pattern 1: broken wave plunges and induces a jet flow that impinges on the platform front and bottom; Pattern 2: highly-breaking wave with the 


\begin{tabular}{lll}
\hline Water depth & $d$ & $0.80 \mathrm{~m}$ \\
Number of wave components & $N$ & 32 \\
Frequency band & {$\left[f_{\min }, f_{\max }\right]$} & {$[0.32 \mathrm{~Hz}, 0.96 \mathrm{~Hz}]$} \\
Amplitude of the $i$-th component & $a_{i}$ & $0.0068 \mathrm{~m}$ \\
Frequency of the $i$-th component & $f_{i}$ & Uniformly discretised in the frequency \\
band & Computed by the dispersion equation \\
Characteristic wave frequency & $f=\left(f_{\min }+f_{\max }\right) / 2$ & $0.64 \mathrm{~Hz}$ \\
Characteristic wave length & $L$ & $3.43 \mathrm{~m}$ \\
Characteristic wave celerity & $C$ & $2.19 \mathrm{~m} / \mathrm{s}$ \\
\hline & & $11.800 \mathrm{~m} / 20.360 \mathrm{~s}$ \\
& & $11.900 \mathrm{~m} / 20.420 \mathrm{~s}$ \\
& & $11.950 \mathrm{~m} / 20.450 \mathrm{~s}$ \\
& & $11.975 \mathrm{~m} / 20.465 \mathrm{~s}$ \\
Focusing position/time & & $12.000 \mathrm{~m} / 20.480 \mathrm{~s}$ \\
& $x_{\mathrm{f}} / t_{\mathrm{f}}$ & $12.025 \mathrm{~m} / 20.490 \mathrm{~s}$ \\
& & $12.050 \mathrm{~m} / 20.500 \mathrm{~s}$ \\
& & $12.100 \mathrm{~m} / 20.520 \mathrm{~s}$ \\
& & $12.200 \mathrm{~m} / 20.580 \mathrm{~s}$ \\
& &
\end{tabular}

\section{Results and discussion}

Fig. 4 shows the wave elevation at $1.438 \mathrm{~m}$ in front of the platform for the case d80xf12000. Due to the wave focusing, a large wave crest of $0.192 \mathrm{~m}$ appears suddenly, forming a freak wave. The wave elevation data of two repeated runs coincide very well. The good repeatability indicates the reliability of the wave generation. After passing through the wave gauge, the freak wave can further develop and

tongue impacting the front wall of the deck; Pattern 3: highly-breaking wave with the tongue impacting the top wall of the deck; Pattern 4: slightly- or non-breaking wave still developing

Table 1 Summary of parameters for wave generation 


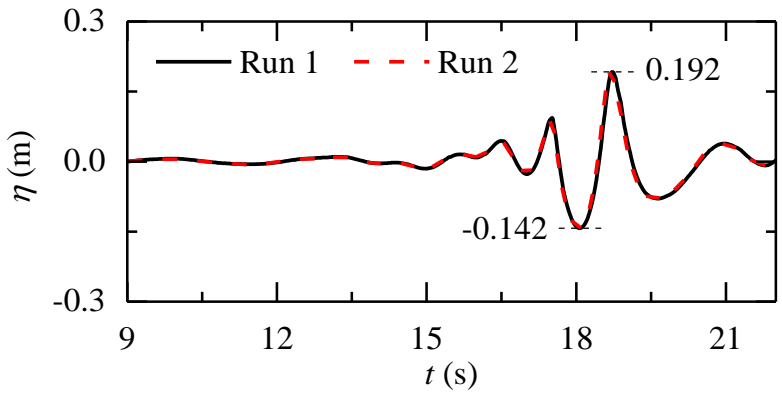

Fig. 4. Wave elevation of the case d80xf12000 measured at a location $1.438 \mathrm{~m}$ in front of the platform

261 The wave profile just in front of the structure varies with the focusing location of the wave packet.

262 With a small $x_{\mathrm{f}}$, the freak wave may get broken before reaching the structure. With the increase of $x_{\mathrm{f}}$, 263 the freak wave may just break (see Fig. 5a and b) or still develop (see Fig. 5c) upon the wave impact 264 happens. Although the characteristics of the wave-structure interaction vary with the incident wave 265 profile, they show some common features. When the wave arrives at the pontoon, the wave diffraction 266 and shoaling happen, which cause the amplification of wave height and the decrease of wave velocity 267 [49-51] (see Fig. 6a). The waves in the gap between the two pontoons and those between the pontoons 268 and the sidewalls keep going in the constant water depth region. Consequently, the wave velocity decreases from the flume centre line to the lateral sides of the platform and then increases in the side gaps between the pontoons and the sidewalls. Such velocity distribution produces a W-shape wave front along the flume width direction that can be seen from Fig. 6b. The ' $W$ ' shape can be further verified by the starting time of the wave impact pressures on FP1, FP3 and FP4, among which FP3 that locates in the middle of the front front wall records wave pressure the earliest. Note that the waves above the pontoons, although with a lower celerity, break earlier because of the shoaling effect. The breaking waves surge forward and impact on the port and starboard sides of the front wall (FP4 is here) at an earlier time than the wave impact at the quarter position of the front wall (FP1 is here). The plungingtype freak wave together with the ' $W$ ' shape wave front leads to an air entrapment zone near the quarter position of the front wall. Fig. $6 \mathrm{c}$ shows the escape of the air entrapment clearly. The wave impact pressure within this region (FP1) shows oscillations because of the vibration of the air pocket.
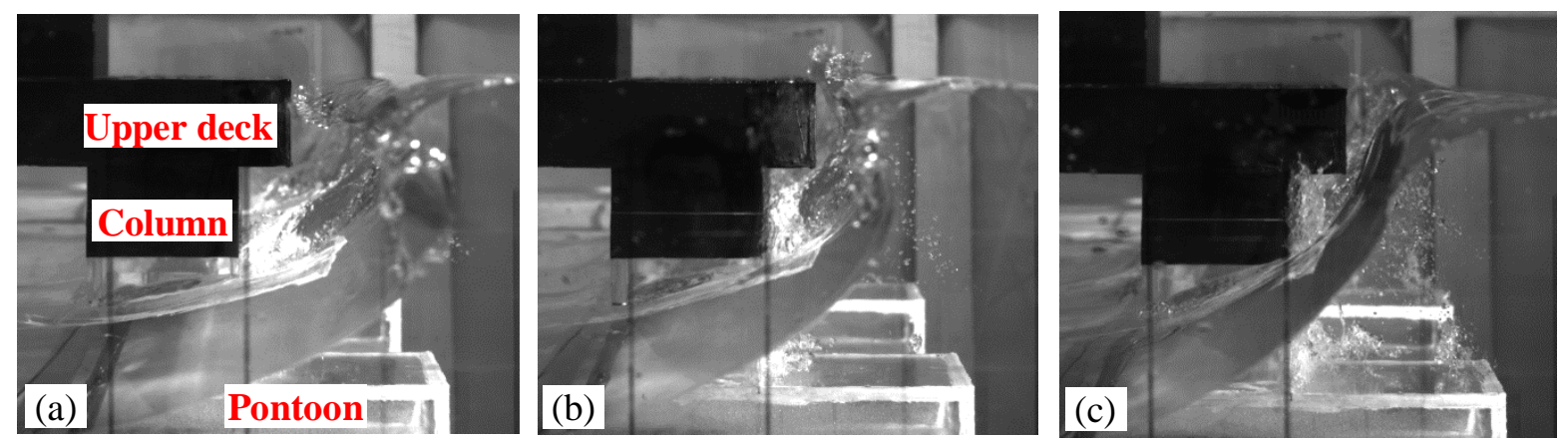

Fig. 5. Wave profile snapshots of typical cases: (a) d80xf11800; (b) d80xf12000; (c) d80xf12300 
The gauge pressures on the measurement locations are normalized by $\rho C^{2}$, where $\rho$ is the water density $\left(1000 \mathrm{~kg} / \mathrm{m}^{3}\right)$ and $C$ the typical wave celerity corresponding to the characteristic wave frequency (see the numbers in Table 1).

\subsubsection{Front vertical wall}

The wave impact pressures with different wave focusing locations are presented in Fig. 7. The front wall is under very violent wave actions. FP1 in all the cases shows pressure oscillations. The oscillations manifest several regular cycles that correspond to the compression and expansion of the entrapped air pocket, and the decay of the amplitude is slower. Specifically, the rise time of the first pressure peak is around 5 millisecond and the period of an oscillation cycle is around 10 millisecond. The 'subatmospheric' phenomenon $[13,52]$ is observed with the amplitude of negative pressure reaching $1 \rho C^{2}$. These phenomena are analogous to those of the high-aeration impact presented in [13] although that study reported a much longer rise time of around 30 milliseconds. The difference in the recorded rise time may be explained by the correlation between the oscillation frequency of an air pocket and its volume as reported in [41]. The pressure signal of FP1 is characterized by large peak values. For the highly-breaking wave cases of impact patterns 2 and 3 , the peak is within the range of $3.5 \rho C^{2}$ to $5 \rho C^{2}$. The two cases in wave pattern 4 show a decrease of the peak with the downstream shift of the wave focusing location. It is anticipated that the trend applies with a further increase of $x_{\mathrm{f}}$ as waves become less developed when impacting on the structure. The large peak and high-frequency oscillation of the impact pressure in the air entrapment region may cause cavitation damages to the structure [52]. It should be noted that the dynamics of the entrapped air does not follow the Froude scaling well and should be considered in the scaling of the results of laboratory studies to prototype problems $[53,54]$.
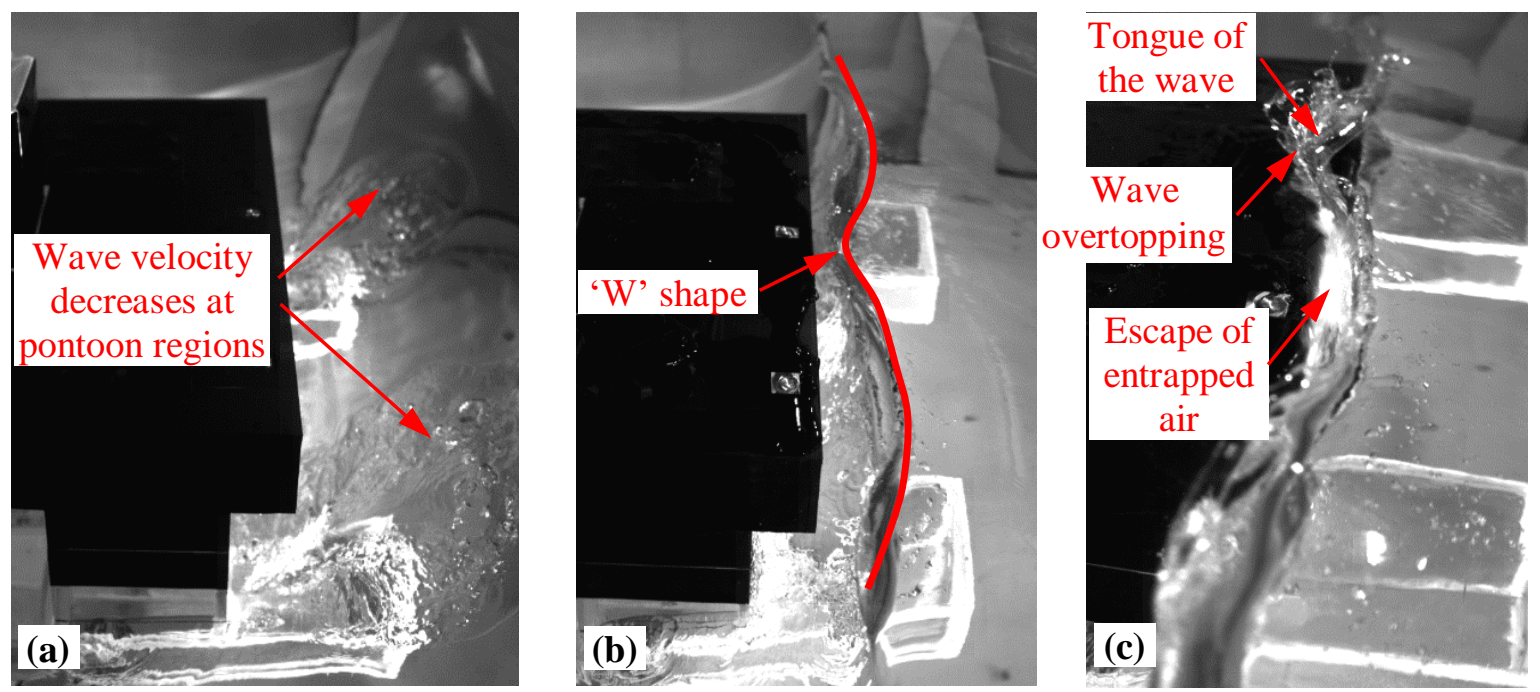

Fig. 6. Aerial view of the wave profiles in case d80xf12000: (a) wave shoaling at the pontoon regions; (b) ' $\mathrm{W}$ ' shape of the wave front; (c) escape of the entrapped air near the quarter location of the front wall 
FP2 and FP3 are located in the middle of the width of the front wall and around 1/3 and 2/3 of the

303 height from the deck bottom. For wave patterns 2 and 3 involving a mature plunging breaker, the tongue

304 of the freak wave has a larger velocity than the main body of the wave and hence impinges on the top

305 front corner of the upper deck first (see Fig. $6 \mathrm{~b}$ and c). This is why FP3 records the impact pressure at

306 the earliest time among the measurement locations and shows a peak. At this time instant, the wave

307 does not touch the bottom part of the front wall yet and hence no impact pressure is recorded on FP2.

308 As the wave propagates, the cavity surrounded by the plunging jet disappears and the main body of the

309 freak wave impacts on the entire front wall, inducing a second peak on FP3 and a large impact pressure

310 on FP2. In general, the signal of FP3 has a long duration and relatively small magnitude $\left(\sim 2 \rho C^{2}\right)$, while

311 that of FP2 behaves more like a pulse with a larger peak $\left(\sim 4.5 \rho C^{2}\right)$ and a short duration.

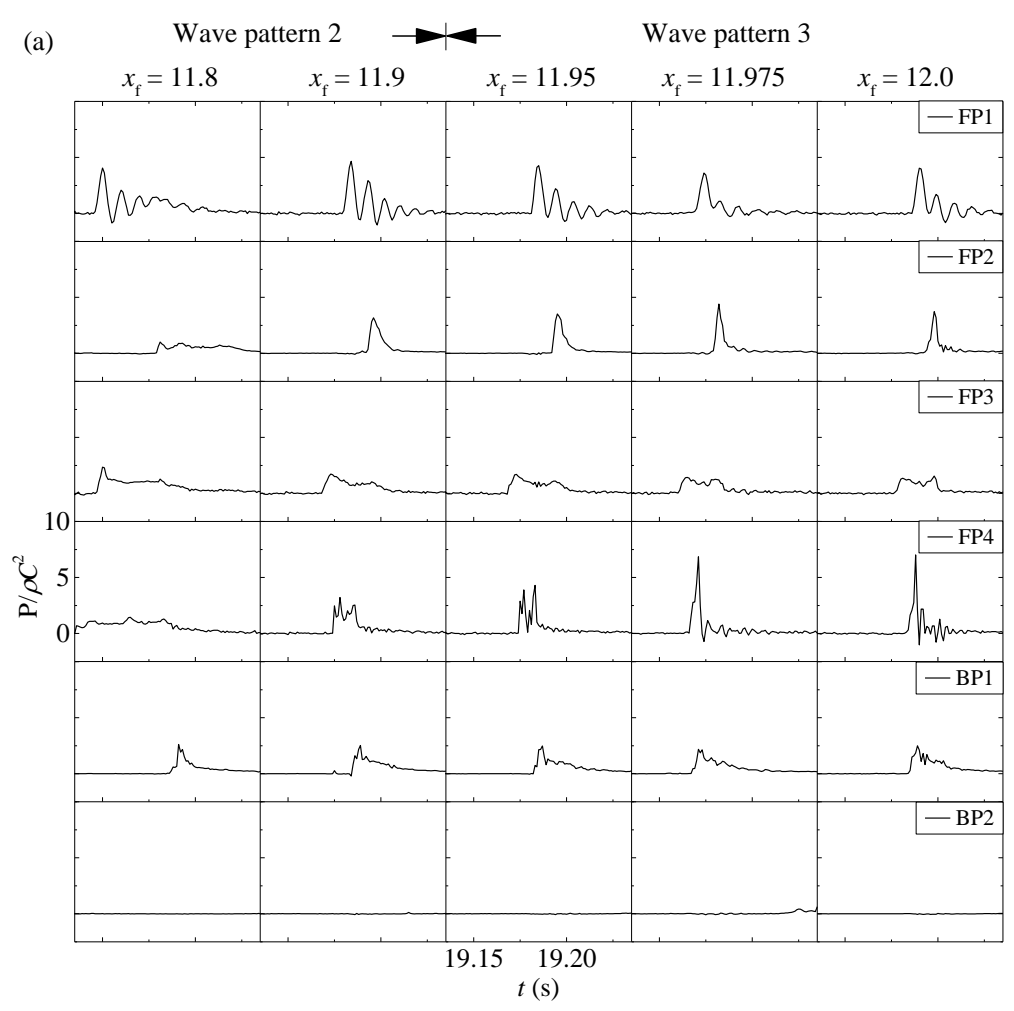




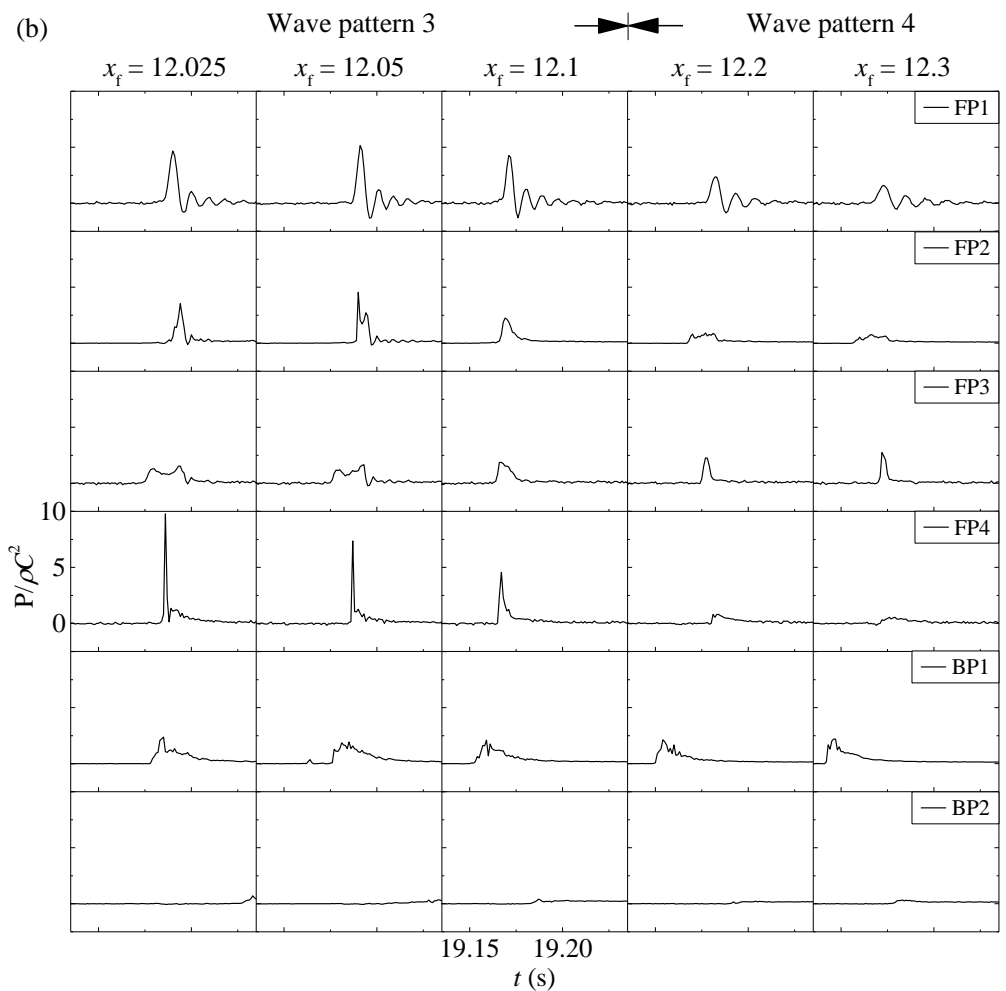

Fig. 7. Variation of pressures on the front and bottom walls of the upper deck with $x_{\mathrm{f}}$

FP4 is very sensitive to the incident wave profile. In general, the impact peak increases and then decreases with the downstream shift of the wave focusing location, while the impact duration correlates with the focusing location in the opposite way. Particularly for the case recording the maximum peak $\left(x_{\mathrm{f}}=12.025 \mathrm{~m}\right.$ at wave pattern 3 that involves the highly-breaking wave), the peak value reaches $10 \rho C^{2}$, the pressure rise time is 1.2 millisecond and the pressure peak decays very quickly. It means that the wave impact on FP4 in this particular case is the low-aerated impact. Indeed, this is the largest impact pressure recorded in all the wave cases. Note that four sensors were installed at selected locations on the front wall, which might not have captured the local maximum pressure.

\subsubsection{Bottom wall}

For the pressures on the bottom wall, BP1 that is near the port side records considerable wave impacts, while the impact pressures in the middle (BP2) are very small. This is because near the port side, after the wave above the pontoon impacts on the front surface of the columns, it turns up and impinges on the bottom of the protruding part of the deck. The peak values of BP1 for all the studied cases locate from $2 \rho C^{2}$ to $3 \rho C^{2}$. On the other hand, the wave skims over the middle of the width direction of the platform, inducing small impact pressures.

Massive wave overtopping happens on the top wall (see Fig. 6c), but do not induce significant wave impacts (hence the pressures at TP1 and TP2 are not presented). Particularly, the pressure maxima are less than $0.5 \rho C^{2}$ for all the cases. This indicates that the impact load induced by green water on the top 
334 of the upper deck is not a serious issue. Instead, the main concern is the influence of the green water on

335 the serviceability of the devices on the deck.

\section{3.1.4. Further discussion on wave impact pressures}

337 Due to the large wave height and steepness, the freak wave impinged upon the platform practically 338 as a wall of water, inducing violent impacts on the front vertical wall facing the incident wave [55].

339 Based on the pressure sensor data collected, the maximum pressure reached $10 \rho C^{2}$ on the front wall and

$3403 \rho C^{2}$ on the bottom wall. On the top wall, significant wave overtopping was observed though it did not 341 cause high impact pressure with a maximum of $0.5 \rho C^{2}$.

342 The impact pressure on the front wall was not spatially uniform. Specifically, the front wall 343 registered larger impact pressure near the port/starboard side due to the diffraction and amplification 344 effects of pontoons on the incident wave. Based on the measurements, the maximum pressure on the 345 front wall (FP4 near the starboard side) is $10 \rho C^{2}$, which is 5 times larger than that in the middle (FP3, 346 at the same elevation as FP4).

\section{3.2. Platform motion}

348 The wave impact on the platform causes rigid body motions of the structure. To illustrate this, the measured results of the case d80xf12000 are presented in Fig. 8. Since the entire experimental setup and waves were symmetrical about the centre line of the wave flume, the sway, yaw and roll motions of the structure were not evident. Therefore, only the surge, heave and pitch motions are presented. Before the main crest of the freak wave comes, the platform is offset towards the up-wave direction (see the second plot of Fig. 8). Under the action of the incident wave, the platform starts to move towards the down-wave direction at $t_{1}=19.037 \mathrm{~s}$ (see Fig. 9 for the wave profile snapshot). Followed, the surge velocity of the platform increases rapidly and reaches the maximum (around $0.8 \mathrm{~m} / \mathrm{s}$, see the third plot of Fig. 8) at $t_{2}=19.213 \mathrm{~s}$. This is the time when the freak wave impingement on the upper deck finishes (see the dropping of the impulse peak on FP2 shown in the top plot of Fig. 8). After that, the incident wave continues applying forces onto the platform, and at the same time, the buoyancy force (resistance force) that the structure is subjected to increases. Hence, the surge motion velocity does not increase further and maintains for some time, during which the platform undergoes a large surge motion. As can be seen in the second plot of Fig. 8, the platform sets down while moving horizontally. The freak wave impingement is transient, which lasts to $t_{3}$. In this process, the motion and tether responses are mainly caused by wave impact, because the plunger breaker of the freak wave applies an intensive impact force over a short duration, which dominates other wave-structure interaction forces (e.g. wave diffraction and radiation forces). After the passage of the freak wave, the wave-structure interaction forces may become the dominating factors affecting the platform motions and tether responses. 


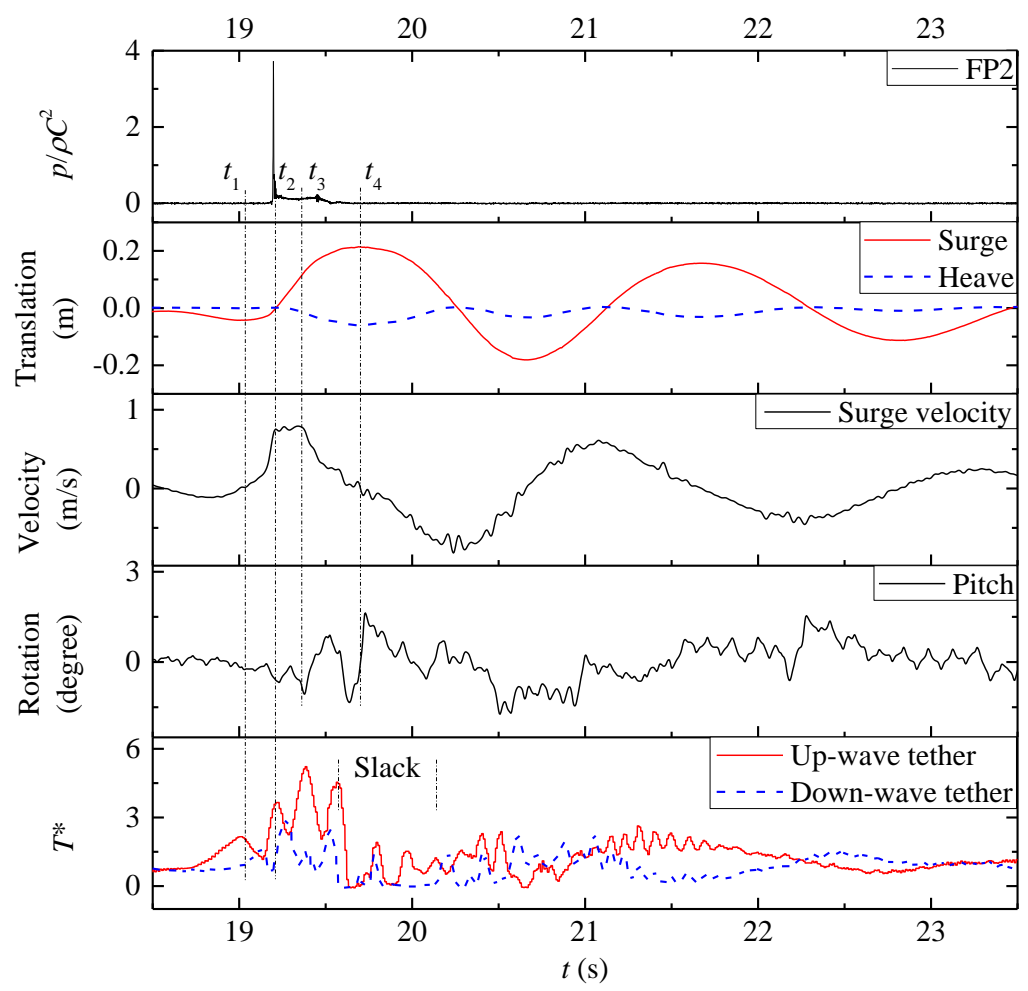

Fig. 8. Wave impact pressure on FP2 (top plot), translational motion (second plot), surge velocity (third plot), pitch motion after a low-pass filter with a cut-off frequency of $22 \mathrm{~Hz}$ (fourth plot), and tether forces (bottom plot) for the case d80xf12000

After $t_{3}=19.36 \mathrm{~s}$, the wave impact becomes small and hence the surge velocity starts to reduce. However, the platform keeps surging downstream and going down under inertia. Up to a certain point, the cables slack, which can be seen clearly from the zero tether force in the bottom plot of Fig. 8. At $t_{4}$ $=19.62 \mathrm{~s}$, the platform gets the maximum surge motion of $0.21 \mathrm{~m}$, which is almost 2 times of that observed in [37]. After that, the platform surges upstream (toward the up-wave direction) and the tethers get re-tensioned. Note that the sudden onset of re-tension after the slack can induce damages to the tethers and ancillary facilities, which is highly undesirable in practical applications $[33,56]$. The platform will then surge back and forth until the kinetic energy of the platform is completely damped. The duration of one motion cycle is around $2.12 \mathrm{~s}$, being much larger than the wave impingement duration.

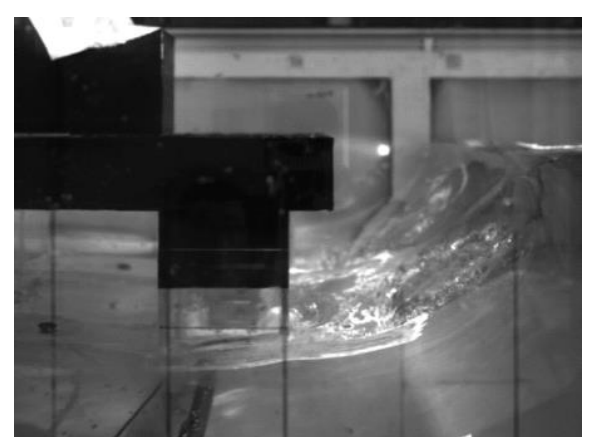

$t_{1}=19.037 \mathrm{~s}$

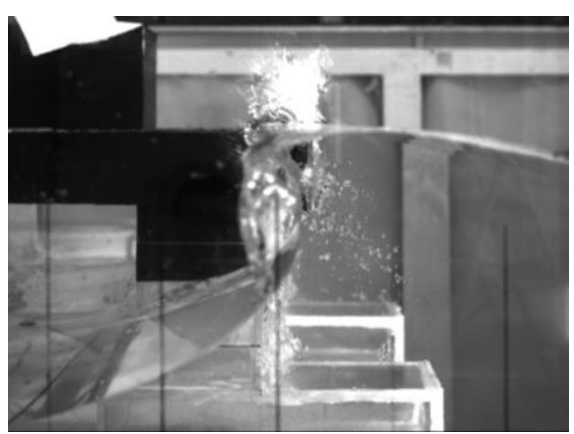

$t_{2}=19.213 \mathrm{~s}$ 


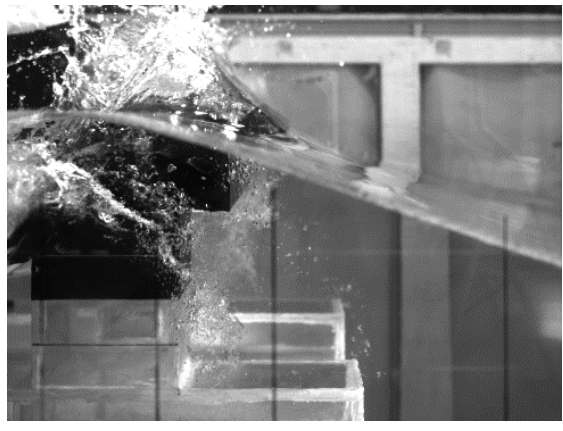

$t_{3}=19.360 \mathrm{~s}$

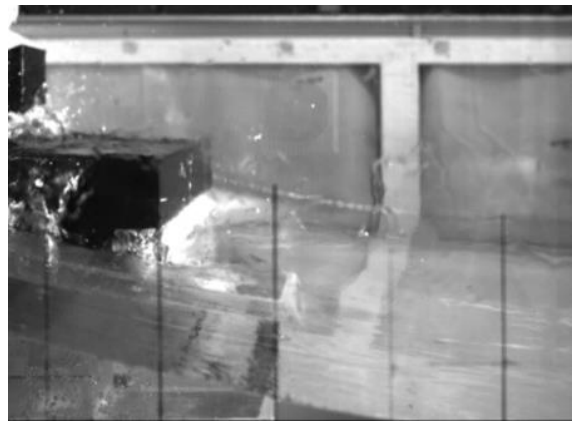

$t_{4}=19.692 \mathrm{~s}$
381

Fig. 9. Wave profile snapshots at four typical time instants (i.e. $t_{1}, t_{2}, t_{3}$ and $t_{4}$ indicated in Fig. 8) for the case d80xf12000

The maximum pitch recorded is $1.655^{\circ}$, which occurs at a time close to that of the maximum surge. After that, the impact wave load disappears and the platform undergoes 'decayed free vibration' approximately (not exactly, because wave-structure interaction forces exist). The accompanied pitch motion manifests small-amplitude fluctuations. A spectrum analysis of the pitch signal at this stage shows dominant frequencies of $7.05 \mathrm{~Hz}, 14.13 \mathrm{~Hz}$ and $21.17 \mathrm{~Hz}$. Based on the decayed free vibration response, it is inferred that the fluctuations in pitch are induced by the ringing phenomenon at $7.05 \mathrm{~Hz}$, which is the pitch natural frequency of the floating platform. The other two frequencies are the integral multiples of the pitch natural frequency. The spectrum analysis also suggests that the raw data of pitch motion contain high-frequency noises and measurement errors. Although steps were taken to minimize noises/errors, it was more difficult to accurately measure small rotational motions (lower signal-tonoise ratio) than translational motions. Therefore, a low-pass filter with a cut-off frequency of $22 \mathrm{~Hz}$ was applied to the pitch signal, and the fourth plot of Fig. 8 shows the filtered pitch result.

The influence of incident wave pattern on platform motion is studied. As shown in Fig. 10, the mean and standard deviation of surge motion are $0.216 \mathrm{~m}$ and $0.002 \mathrm{~m}$, respectively, for the studied cases that cover three impact patterns. Although the surge motion generally does not vary too much with the wave focusing location $x_{\text {f }}$, it shows a trend that the surge motion in wave pattern 2 and 4 is larger than that in wave pattern 3 . This is because a large amount of water collapses on the upper deck in patter 3 , and hence the horizontal and uplift forces applied onto the platform are smaller. These lead to smaller structural motions and smaller tether forces (will be shown in Section 3.3). The mean and standard deviation of pitch are 2.458 degree and 0.510 degree, respectively. The variation with $x_{\mathrm{f}}$ shows the same trend, but the variation is relatively larger. 


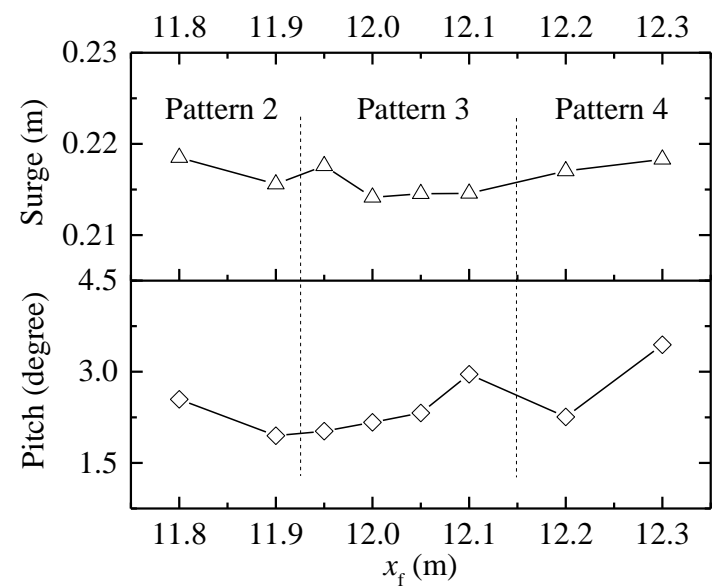

Fig. 10. Variation of platform surge (top plot) and pitch (bottom plot) with incident wave pattern

\subsection{Tether force}

Large forces are generated in the tethers during the wave-structure interaction process. For better illustration, the tether forces are normalized by the initial tether pretention or static force $T^{0}(68.6 \mathrm{~N})$ and denoted by $T^{*}$. As shown in the bottom plot of Fig. 8, in the case d80xf12000 the up-wave and down-wave tethers are subject to forces of 5.2 and 2.9 times of the pretension, respectively. The forces in tethers also exhibit oscillations, with the local peaks of both tether forces being almost 180 degree out of phase. This implies that the oscillations are related to the pitch motion of the platform [57].

Another important feature of the tether force is the sudden drop/increase and even the sudden retention from slack. To quantify this, the dynamic tension, i.e. the range from a local minimum to the immediately following maximum [58, 59], is evaluated. Following the definition in $[59,60]$, a snap event occurs when the dynamic load is more than $90 \%$ of the static load (pretension in tethers). Ten snap events are identified in the up-wave tether force of the case d80xf12000, as represented by the blue dot-dash lines in the top plot of Fig. 11. As can be seen, the dynamic tension (see the bottom plot of Fig. 11) reaches 3 times of the pretension in the first cycle of platform surging downstream. Three local peaks are observed in the dynamic tension curve. Each peak occurs near the time when the platform undergoes in the local maximum (positive or negative) velocity (see the third plot of Fig. 8). This implies that the violent platform motion induces snap events. In the study of mooring tensions of a floating wind turbine under survival sea states [59], an average of 32 out of around 1000 tension-force cycles was identified as snap events. The frequency of occurrence of the snap event is much lower than that induced by a freak wave as studied in the present work. This is mainly because the high-crest freak wave can cause violent motions of the platform. The dynamic loads increase the risk of tether damage and aggravate the fatigue issue of tethers. The DNV standards recommend that the snap events should be avoided to the maximum extent [60]. 


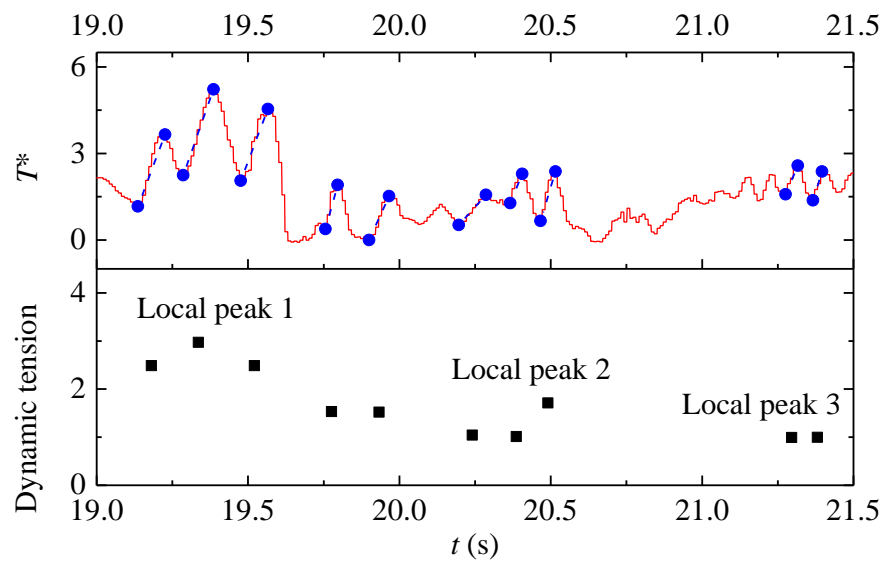

Fig. 11. Snap events in the case d80xf12000

The influence of incident wave pattern on the normalized tether forces is studied and presented in 432 Fig. 12. In general, both tethers withstand large forces in the tested freak wave scenarios. The forces 433 range 5.0 - 6.2 times of the pretension for the up-wave tether and 2.7 - 5.1 times for the down-wave 434 tether. The force in the up-wave tether decreases with the transition from wave pattern 2 to pattern 3 and keeps almost constant (around 5) within wave pattern 3. The force increases again with the transition to wave pattern 4 . The down-wave tether force follows the same trend, but with a smaller amplitude. This leads to that the total force in the two cables is smaller in wave pattern 3 and bigger in wave patterns 2 and 4 . It means that the slightly- or non-breaking waves in wave pattern 4, although applying relatively small wave impact pressures on the deck, induce large tension forces in the tethers.

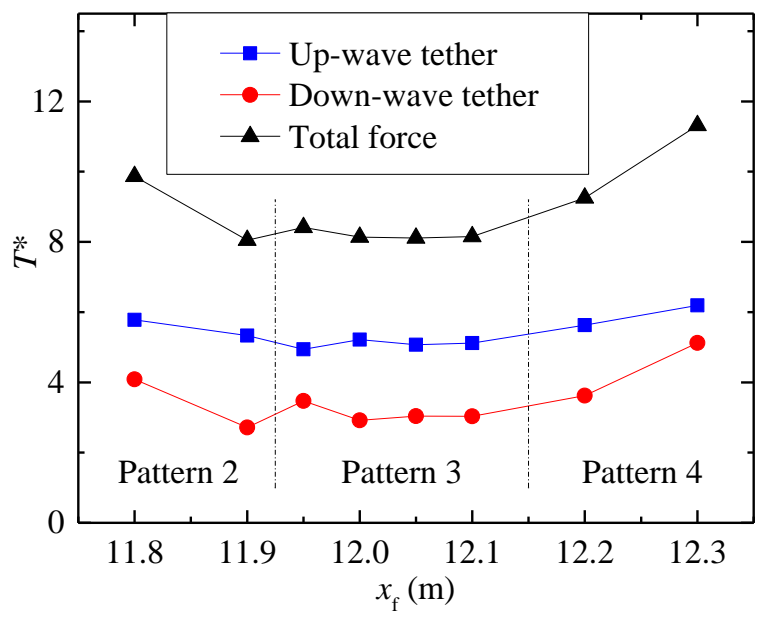

Fig. 12. Variation of normalised tether forces with incident wave pattern

\section{Repeatability analysis of the measurement data}

The impact pressure induced by breaking waves (a major effect of the impinging freak wave) is very sensitive to the wave profile just before the impact happens. Tiny experimental errors that cannot be avoided will be amplified during the generation of a large-amplitude freak wave. This combined with the high nonlinearity of the entrapped air dynamics leads to the variations of impact pressures for 
447 different repeats of the same case with identical inputs. The repeatability issue of breaking wave impacts

448 was also reported in previous studies [6, 35, 37, 39].

449 To test the repeatability and hence the quality of the experimental data, the studied cases were 450 repeated for several runs. For each run, measures were taken to ensure the same experimental condition 451 such as the same water level and the water and structure being visibly stationary before wave generation. 452 As shown in Fig. 4, the wave elevations at $1.438 \mathrm{~m}$ upstream of the upper-deck (see Fig. 1) measured 453 in two repeated runs are very close, although some tiny discrepancies exist. After passing through the 454 wave gauge, the wave packet starts to focus, during which the tiny discrepancies are amplified. This 455 leads to magnified variations in wave profiles and kinematics just in front of the platform. These affect 456 the local wave velocity and the amount of entrapped air upon impact on the structure and hence the 457 characteristics of the impact pressures. In general, the pressure on the same location shows some 458 variations in amplitude and phase among different runs. For two specified runs, the variations of 459 pressure on different locations do not follow a consistent rule.

460 For illustration, the results of the case d80xf12000 are discussed. The wave impact pressures of four 461 runs are presented in Fig. 13 with the statistics of peak pressures listed in Table 2. BP2 is omitted 462 because its value is very small. FP1 is characterized by pressure oscillations. The first cycle captured in 463 runs $1-3$ is quite close including the pressure peak, while that in run 4 shows an evidently smaller 464 amplitude. The maximum coefficient of variation (CV) of the pressure peaks is $21.6 \%$. For the 465 subsequent cycles, the oscillation period is close in different runs, but the decay rate shows some 466 differences. The shape and magnitude of pressure signal FP2 are quite close (with a CV of $8.5 \%$ for the 467 magnitude), but there is a phase delay for run 2. FP3 in the four runs shows a close agreement with the $468 \mathrm{CV}$ of the pressure peak being 9.7\%. FP4 also shows pressure oscillations and evident variations are 469 observed among the repeats, including the occurrence time and value of the first peak (CV of $11.0 \%$ ), 470 the oscillation period and the decay rate. This is because the wave impact on FP4 in this case is the low471 aerated broken wave impact. The air entrapment and the breaking wave introduce uncertainties. BP1 472 shows $20.8 \%$ difference in amplitude and a phase lag as that in FP2. Although the impact pressures 473 differ more or less in repeats of a nominally identical case, the general trend is consistent. 

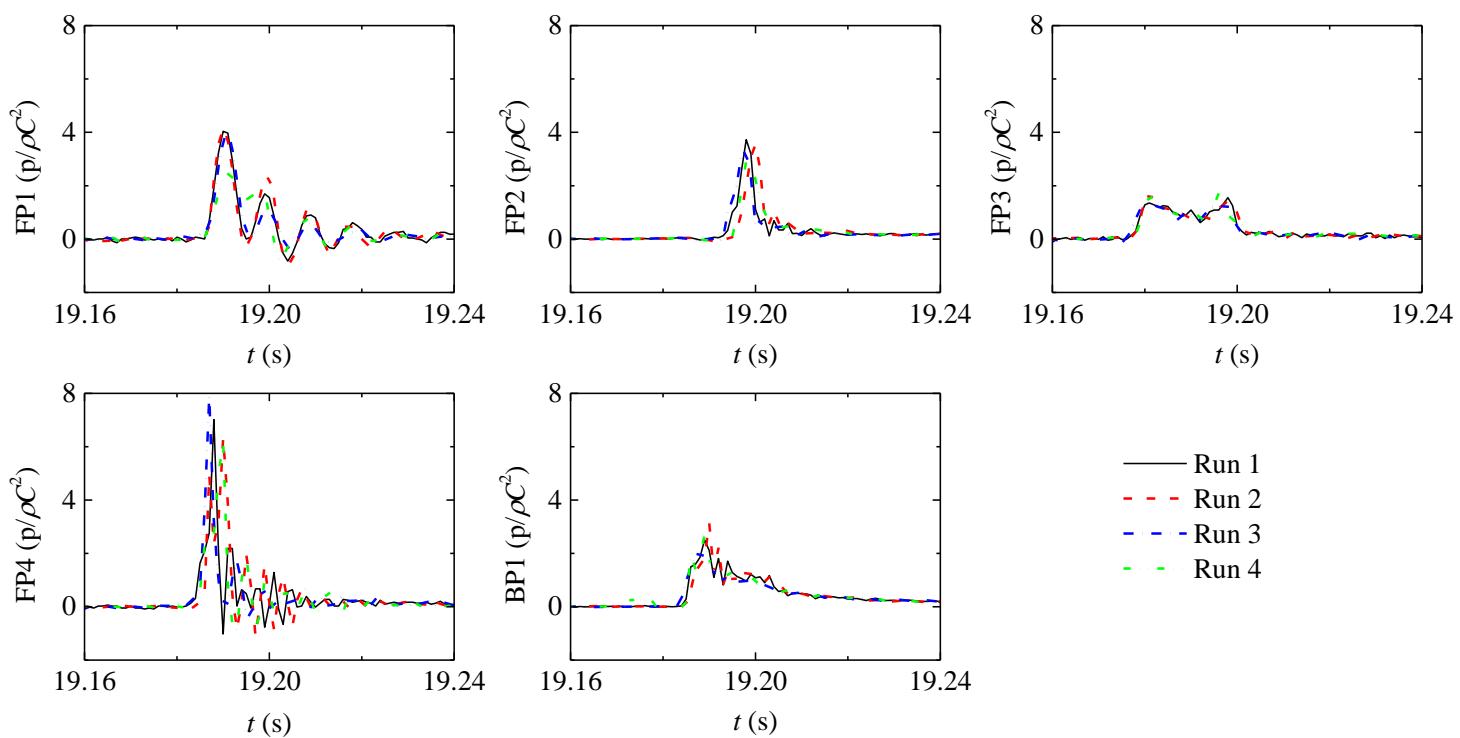

Run

- - Run 2

- - - - Run 3

-.... Run 4

474

475

476

\begin{tabular}{cccccc}
\hline Run id & FP1 $\left(p / \rho C^{2}\right)$ & FP2 $\left(p / \rho C^{2}\right)$ & FP3 $\left(p / \rho C^{2}\right)$ & FP4 $\left(p / \rho C^{2}\right)$ & BP1 $\left(p / \rho C^{2}\right)$ \\
\hline 1 & 4.041 & 3.727 & 1.552 & 7.017 & 2.502 \\
2 & 4.137 & 3.543 & 1.700 & 6.322 & 3.135 \\
3 & 4.035 & 3.270 & 1.383 & 7.902 & 2.071 \\
4 & 2.485 & 3.075 & 1.710 & 6.282 & 2.674 \\
Mean & 3.674 & 3.403 & 1.586 & 6.881 & 2.595 \\
$\sigma$ & 0.794 & 0.289 & 0.154 & 0.760 & 0.440 \\
CV $(\%)$ & 21.6 & 8.5 & 9.7 & 11.0 & 16.9 \\
\hline
\end{tabular}

Fig. 13. Wave impact pressures in four repeated runs of the case d80xf12000

Table 2 Peak pressure measured in four repeated runs of the case d80xf12000
477

478

479

480

481

482

483

484

485

486

The surge and pitch motion histories are presented in Fig. 14 with the motion amplitude tabulated in Table 3. The heave motion is not given because it is correlated with the surge motion and hence has similar repeatability. As can be seen, the surge motion shows a good repeatability with a CV of $1.0 \%$. In contrast, the pitch motion does not repeat well among different runs. This is due to the complexity of the pitch motion (coupling with ringing) and the difficulty of the motion capture system to measure it. Specifically, the CV of the peak value of pitch is $26.3 \%$. The results of tether forces are presented in Fig. 15 and Table 3. All the runs capture similar results in terms of force peaks and fluctuations. The CVs of up-wave and down-wave tether forces are $3.3 \%$ and $3.9 \%$, respectively. The force fluctuations in different runs behave the same trend with the local peaks varying a little bit. 

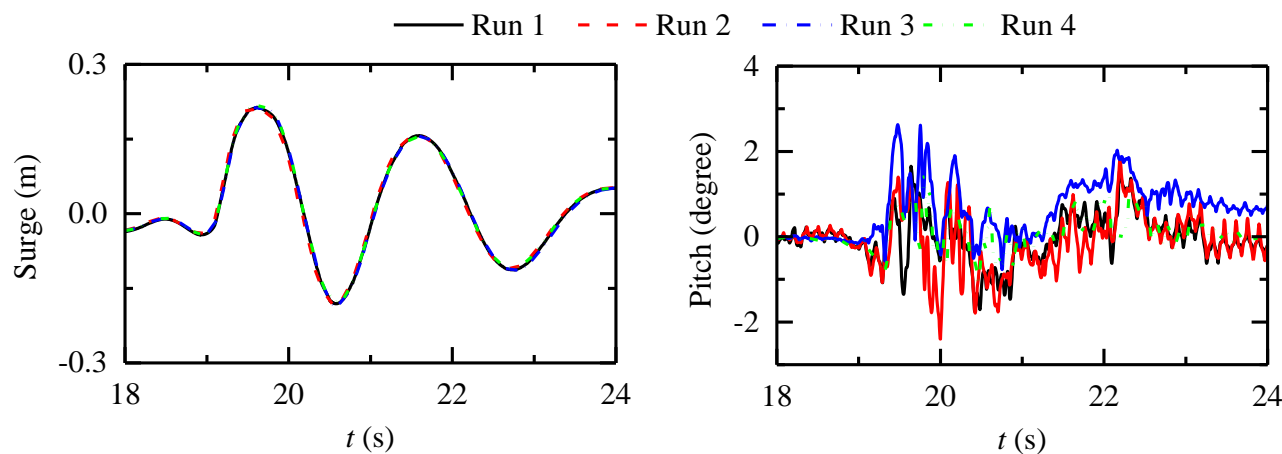

487

Fig. 14. Surge and pitch motions of four repeated runs in the case d80xf12000
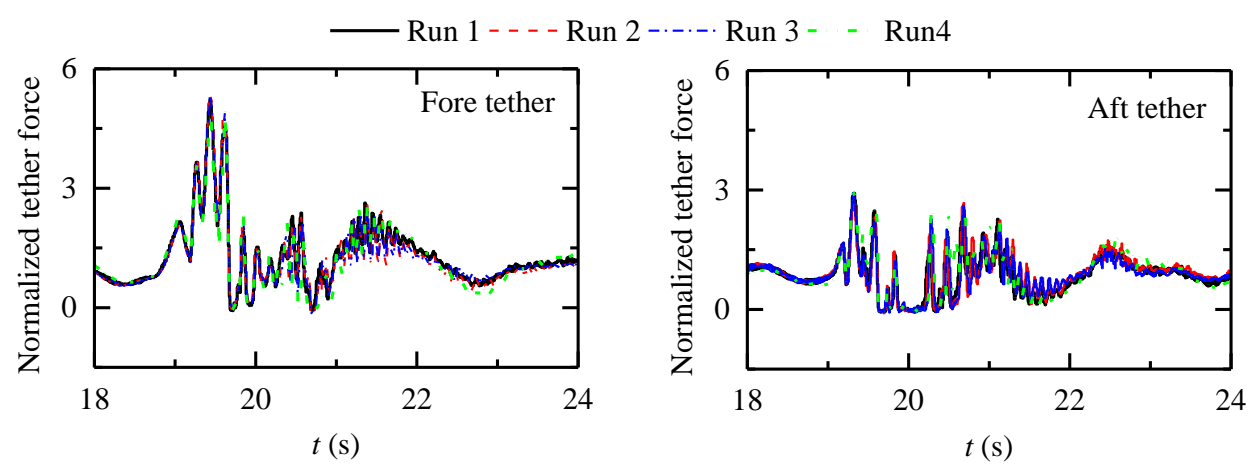

489

Fig. 15. Forces in up- and down-wave tethers of four repeated runs in the case d80xf12000

491

Table 3 Peaks of motions and tether forces measured in four repeated runs of the case d80xf12000

\begin{tabular}{ccccc}
\hline Run id & Surge $(\mathrm{m})$ & Pitch (degree) & $T_{\text {up }}^{*}$ & $T_{\text {down }}^{*}$ \\
\hline 1 & 0.214 & 1.655 & 5.218 & 2.918 \\
2 & 0.213 & 1.835 & 5.339 & 2.716 \\
3 & 0.218 & 2.627 & 5.337 & 2.927 \\
4 & 0.217 & 1.500 & 4.969 & 2.965 \\
Mean & 0.215 & 1.940 & 5.216 & 2.882 \\
$\sigma$ & 0.002 & 0.501 & 0.174 & 0.112 \\
CV $(\%)$ & 1.0 & 26.3 & 3.3 & 3.9 \\
\hline
\end{tabular}

\section{Conclusions}

This study experimentally investigates the hydrodynamic performance of a TLP model (a new design proposed recently) under freak wave impacts. The significance of this study lies in two aspects. First, a comprehensive measurement of wave kinematics/dynamics, structural motions and tether forces was conducted. The experimental results are useful for in-depth analysis and benchmarks (rarely documented in the literature) for validating numerical and analytical models. Second, a synchronous analysis of multi-sensor data is carried out to examine the hydrodynamic performance of the TLP model under a freak wave that has similar properties to the Draupner wave. The analysis shows the destructive actions of freak waves to the tension-leg moored platform. Specifically, the water-wall impinging on structure applies large impact pressures with amplitudes reaching $10 \rho C^{2}$ and the resulting intensive 
502 loads further cause large platform offset and tether forces (six times of the pretension). Two of the

503 findings deserve highlighting. (1) Although applying less intensive local impact pressures as compared

504 to the highly-breaking freak wave (patterns 2 and 3), the slightly-breaking or non-breaking freak wave

505 (wave pattern 4) imposes the same level of adverse effect on the global stability of the floating platform

506 in terms of platform motions and tether forces. (2) The high-crest freak wave causes violent motions of

507 the floating platform, which further induce snap loads of large amplitude and high occurrence frequency

508 in tethers.

509 Though the wave scenario studied is more severe than typically considered in current design codes,

510 the results would be useful in the context of global climate change and the resulting increase in wave

511 power. The authors are developing a numerical model for the interaction between highly-deformed

512 waves and moving structures. The numerical model will be validated by the experimental results

513 presented herein for severe freak waves and extended to study other water depths and wave conditions.

\section{Acknowledgement}

515 The experimental work was financially supported by the Singapore Maritime Institute and Sembcorp 516 Marine Technology Pte Ltd (research grant SMI-2014-OF-02). The first author appreciates the Open 517 Research Funding SKHL1710 and SKHL1712 from the State Key Laboratory of Hydraulics and 518 Mountain River Engineering at Sichuan University, China.

\section{Appendix A. Data to reproduce the experimental cases in this study}

520 The dimensions of the platform are shown in Fig. A1. The mass of the platform is $62.036 \mathrm{~kg}$, and 521 the centre of gravity locates the geometrical centre when viewed in plan and $0.2182 \mathrm{~m}$ above the bottom 522 of the pontoon when viewed in elevation. The locations of the pressure sensors are detailed in Fig. A2. 523 Other experimental data can be found in Mendeley Data or will be shared upon request by contacting 524 the first author. 


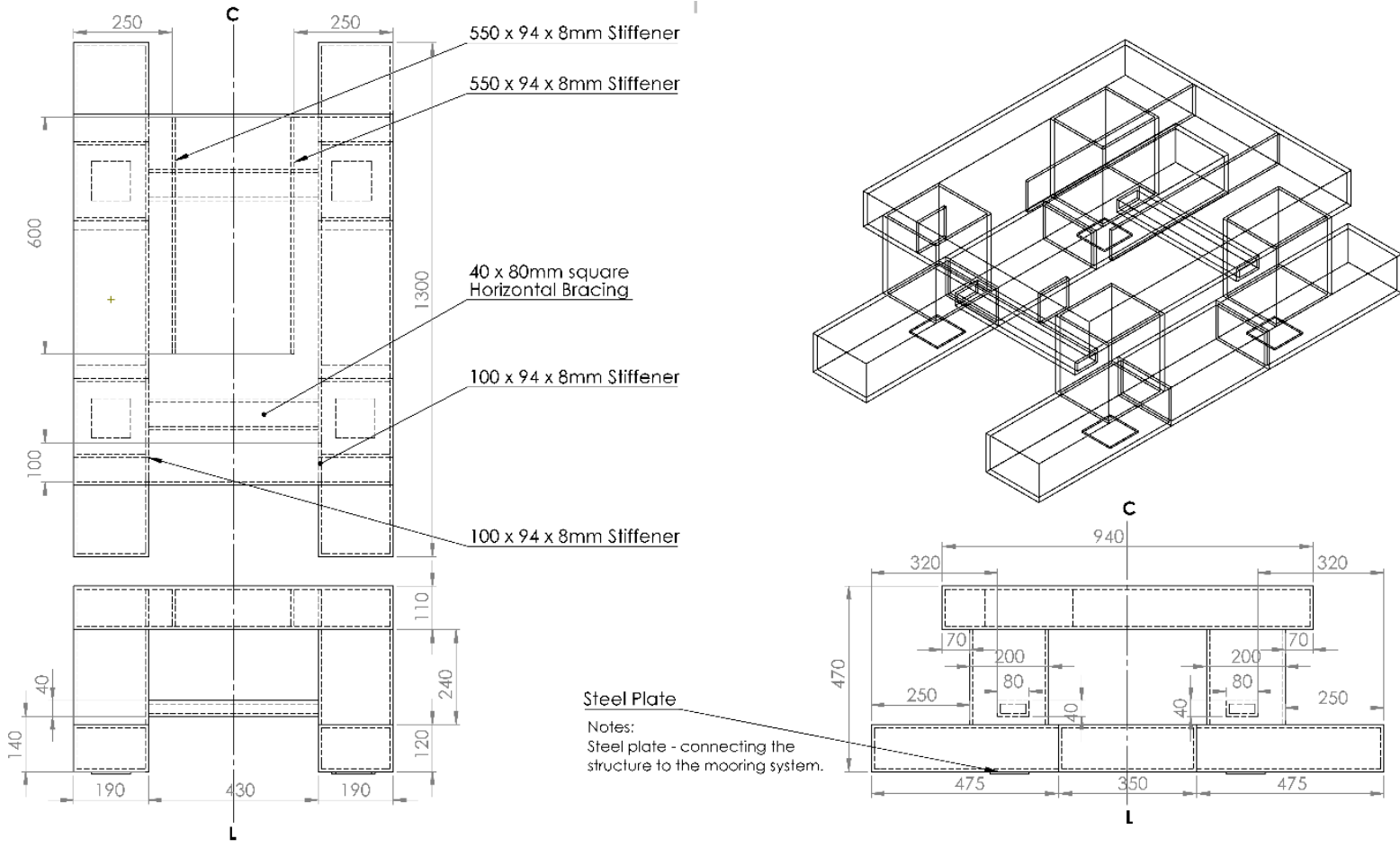

Fig. A1. Dimensions of the floating platform
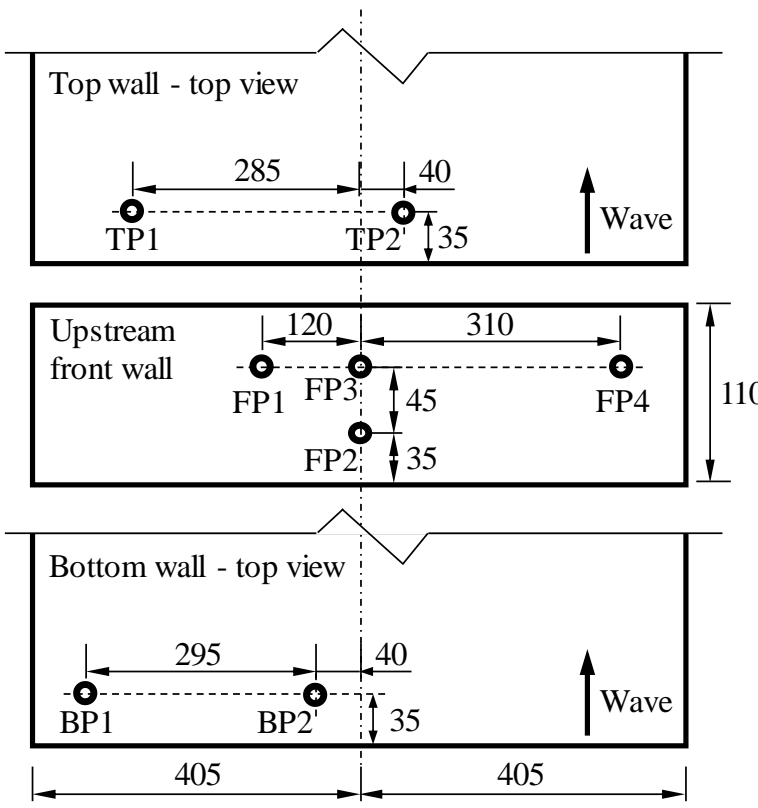

Fig. A2. Locations of pressure sensors (Unit: mm)

\section{References}

530 [1] Dysthe K, Krogstad HE, Müller P. Oceanic rogue waves. Annual Review of Fluid Mechanics. $531 \quad 2008 ; 40: 287-310$.

532 [2] Kharif C, Pelinovsky E. Physical mechanisms of the rogue wave phenomenon. European Journal of 533 Mechanics - B/Fluids. 2003;22:603-34.

534 [3] Fochesato C, Grilli S, Dias F. Numerical modeling of extreme rogue waves generated by directional 535 energy focusing. Wave Motion. 2007;44:395-416. 
536 [4] Karin Magnusson A, Donelan MA. The Andrea Wave Characteristics of a Measured North Sea

537 Rogue Wave. Journal of Offshore Mechanics and Arctic Engineering. 2013;135.

538 [5] Bitner-Gregersen EM, Gramstad O. Rogue waves: Impact on ships and offshore structures. DNV

539 GL Strategic Research \& Innovation: DNV GL; 2016.

540 [6] Chan ES, Melville WK. Deep-water plunging wave pressures on a vertical plane wall. Proceedings

541 of the Royal Society A: Mathematical, Physical and Engineering Sciences. 1988:95-131.

542 [7] Baldock TE, Swan C. Extreme waves in shallow and intermediate water depths. Coastal Engineering. 543 1996;27:21-46.

544 [8] Zhao X, Ye Z, Fu Y, Cao F. A CIP-based numerical simulation of freak wave impact on a floating 545 body. Ocean Engineering. 2014;87:50-63.

546 [9] Yan B, Luo M, Bai W. An experimental and numerical study of plunging wave impact on a box547 shape structure. Marine Structures. 2019;66:272-87.

548 [10] Sun P-N, Luo M, Le Touzé D, Zhang AM. The suction effect during freak wave slamming on a 549 fixed platform deck: Smoothed particle hydrodynamics simulation and experimental study. Physics of $550 \quad$ Fluids. 2019;31:117108.

551 [11] Peregrine DH. Water waves, nonlinear Schrödinger equations and their solutions. The Journal of 552 the Australian Mathematical Society Series B Applied Mathematics. 1983;25:16-43.

553 [12] Chabchoub A, Hoffmann NP, Akhmediev N. Rogue wave observation in a water wave tank. 554 Physical review letters. 2011;106:204502.

555 [13] Bullock GN, Obhrai C, Peregrine DH, Bredmose H. Violent breaking wave impacts. Part 1: Results 556 from large-scale regular wave tests on vertical and sloping walls. Coastal Engineering. 2007;54:602-17.

557 [14] Hu ZZ, Mai T, Greaves D, Raby A. Investigations of offshore breaking wave impacts on a large 558 offshore structure. Journal of Fluids and Structures. 2017;75:99-116.

559 [15] Mai T, Mai C, Raby A, Greaves DM. Aeration effects on water-structure impacts: Part 2. Wave 560 impacts on a truncated vertical wall. Ocean Engineering. 2019;186:106053.

561 [16] Lind SJ, Stansby PK, Rogers BD, Lloyd PM. Numerical predictions of water-air wave slam using 562 incompressible-compressible smoothed particle hydrodynamics. Applied Ocean Research. 2015;49:5756371.

564 [17] Kim N, Kim CH. Investigation of a dynamic property of Draupner freak wave. International journal 565 of offshore and polar engineering. 2003;13.

566 [18] Deng Y, Yang J, Zhao W, Li X, Xiao L. Freak wave forces on a vertical cylinder. Coastal 567 Engineering. 2016;114:9-18.

568 [19] Alagan Chella M, Bihs H, Myrhaug D. Wave impact pressure and kinematics due to breaking wave impingement on a monopile. Journal of Fluids and Structures. 2019;86:94-123.

571 Mathematics. 2004;48:187-217.

572 [21] Qin H, Tang W, Xue H, Hu Z. Numerical study of nonlinear freak wave impact underneath a fixed 573 horizontal deck in 2-D space. Applied Ocean Research. 2017;64:155-68. 

and vertical wave forces on an elevated coastal structure. Coastal Engineering. 2017;128:58-74.

[23] Chen L, Taylor PH, Draper S, Wolgamot H. 3-D numerical modelling of greenwater loading on fixed ship-shaped FPSOs. Journal of Fluids and Structures. 2019;84:283-301.

[24] Hu Z, Tang W, Xue H, Zhang X, Wang K. Numerical study of rogue wave overtopping with a fully-coupled fluid-structure interaction model. Ocean Engineering. 2017;137:48-58.

[25] Chuang W-L, Chang K-A, Mercier R. Kinematics and dynamics of green water on a fixed platform in a large wave basin in focusing wave and random wave conditions. Experiments in Fluids. 2018;59.

[26] Zhao X, Hu C. Numerical and experimental study on a 2-D floating body under extreme wave conditions. Applied Ocean Research. 2012;35:1-13.

[27] Cheng L, Lin P. The numerical modeling of coupled motions of a moored floating body in waves. Water. 2018;10:1748.

[28] Wei Y, Abadie T, Henry A, Dias F. Wave interaction with an Oscillating Wave Surge Converter.

[29] Gonçalves RT, Rosetti GF, Fujarra ALC, Oliveira AC. Experimental study on vortex-induced motions of a semi-submersible platform with four square columns, Part II: Effects of surface waves, external damping and draft condition. Ocean Engineering. 2013;62:10-24.

[30] Xu S, Ji C-y, Guedes Soares C. Experimental and numerical investigation a semi-submersible moored by hybrid mooring systems. Ocean Engineering. 2018;163:641-78.

[31] Deng Y, Yang J, Xiao L. Influence of wave group characteristics on the motion of a semisubmersible in freak waves. The ASME 2014 33rd International Conference on Ocean, Offshore and Arctic Engineering. San Francisco, USA2014.

[32] Rudman M, Cleary PW. Rogue wave impact on a tension leg platform: The effect of wave incidence angle and mooring line tension. Ocean Engineering. 2013;61:123-38.

598 [33] Rudman M, Cleary PW. The influence of mooring system in rogue wave impact on an offshore 599 platform. Ocean Engineering. 2016;115:168-81.

600 [34] Chuang W-L, Chang K-A, Mercier R. Green water velocity due to breaking wave impingement on 601 a tension leg platform. Experiments in Fluids. 2015;56.

602 [35] Chuang W-L, Chang K-A, Mercier R. Impact pressure and void fraction due to plunging breaking 603 wave impact on a 2D TLP structure. Experiments in Fluids. 2017;58.

604 [36] Abdussamie N, Ojeda R, Drobyshevski Y, Thomas G, Amin W. Experimental investigation of 605 extreme wave impacts on a rigid TLP model in cyclonic conditions. Ships and Offshore Structures. $606 \quad 2016 ; 12: 153-70$.

607 [37] Abdussamie N, Drobyshevski Y, Ojeda R, Thomas G, Amin W. Experimental investigation of 608 wave-in-deck impact events on a TLP model. Ocean Engineering. 2017;142:541-62.

609 [38] TheEngineeringToolbox. Young's Modulus - Tensile and Yield Strength for common Materials.

610 [39] Vestbøstad TM, Økland OD, Lian G, Stavang TP. Column slamming loads on a TLP from steep 611 and breaking waves. ASME 2017 36th International Conference on Ocean, Offshore and Arctic 612 Engineering: American Society of Mechanical Engineers Digital Collection; 2017. 
613 [40] Stansberg CT, Berget K, Graczyk M, Muthanna C, Pakozdi C. Breaking wave kinematics and 614 resulting slamming pressures on a vertical column. ASME 2012 31st International Conference on 615 Ocean, Offshore and Arctic Engineering: American Society of Mechanical Engineers Digital Collection; 616 2012. p. 679-89.

617 [41] Luo M, Koh CG. Shared-Memory parallelization of consistent particle method for violent wave 618 impact problems. Applied Ocean Research. 2017;69:87-99.

619 [42] McGovern DJ, Bai W. Experimental study on kinematics of sea ice floes in regular waves. Cold 620 Regions Science and Technology. 2014;103:15-30.

621 [43] McAllister ML, Draycott S, Adcock TAA, Taylor PH, van den Bremer TS. Laboratory recreation 622 of the Draupner wave and the role of breaking in crossing seas. Journal of Fluid Mechanics. $6232018 ; 860: 767-86$.

[44] Pakozdi C, Östman A, Ji G, Stansberg CT, Reum O, Ovrebo S, et al. Estimation of Wave Loads due to Green Water Events in 10000-Year Conditions on a TLP Deck Structure. ASME 2016 35th International Conference on Ocean, Offshore and Arctic Engineering: American Society of Mechanical Engineers Digital Collection; 2016.

[45] Reguero BG, Losada IJ, Mendez FJ. A recent increase in global wave power as a consequence of oceanic warming. Nature communications. 2019;10:205.

630 [46] Morim J, Hemer M, Wang XL, Cartwright N, Trenham C, Semedo A, et al. Robustness and 631 uncertainties in global multivariate wind-wave climate projections. Nature Climate Change. $632 \quad 2019 ; 9: 711-8$.

633 [47] Rapp RJ, Melville WK. Laboratory measurements of deep-water breaking waves. Philosophical 634 Transactions of the Royal Society of London Series A, Mathematical and Physical Sciences. $6351990 ; 331: 735-800$.

636 [48] Liang S, Sun Z, Zhang Y, Shen J, Zhang Y. Laboratory study on the characteristics of deep-water 637 breaking waves. Procedia Engineering. 2015;116:414-21.

[49] Stansberg CT. Nonlinear wave amplification around column-based platforms in steep waves. ASME 2014 33rd International Conference on Ocean, Offshore and Arctic Engineering: American Society of Mechanical Engineers Digital Collection; 2014.

641 [50] Pessoa J, Stansberg CT, Fonseca N, Laranjinha M. Experimental and numerical study of the free 642 surface elevation over the pontoons of a semisubmersible platform in waves. ASME 2018 37th 643 International Conference on Ocean, Offshore and Arctic Engineering: American Society of Mechanical 644 Engineers Digital Collection; 2018.

645 [51] DNVGL. Prediction of air gap for column stabilised units. Offshore Technical Guidance: DNV 646 GL; 2019.

647 [52] Hsiao S-C, Lin T-C. Tsunami-like solitary waves impinging and overtopping an impermeable 648 seawall: Experiment and RANS modeling. Coastal Engineering. 2010;57:1-18.

649 [53] Cuomo G, Shimosako K-i, Takahashi S. Wave-in-deck loads on coastal bridges and the role of air. 650 Coastal Engineering. 2009;56:793-809.

651 [54] Cuomo G, Allsop W, Takahashi S. Scaling wave impact pressures on vertical walls. Coastal 652 Engineering. 2010;57:604-9.

653 [55] DNVGL. Horizontal wave impact loads for column stabilised units. Offshore Technical Guidance: 654 DNV GL; 2019. 
655 [56] Johannessen TB, Haver S, Bunnik T, Buchner B. Extreme wave effects on deep water Tlps lessons 656 learned from the Snorre a model tests. Deep Offshore Technology. Houston, TX2006.

657 [57] Chakrabarti SK. Hydrodynamics of offshore structures: WIT press; 1987.

658 [58] DNV. Position mooring. Offshore standard DNV-OS-E301. Hovik, Norway2008.

659 [59] Hsu W-t, Thiagarajan KP, Manuel L. Extreme mooring tensions due to snap loads on a floating 660 offshore wind turbine system. Marine Structures. 2017;55:182-99.

661 [60] DNV. Modeling and analysis of marine operations. Recommended practice DNV-RP-H103. Hovik, 662 Norway: Det Norske Veritas2011.

663 